\title{
Study to evaluate effect of high fat Indian meal on postprandial lipid proffle in type 2 diabetics from Central India
}

K. Gupta', S. Vali', S. Gupta ${ }^{3}$.

'Sunil's Diabetes Care n' Research Centre Pvt Ltd, Dietetics and Therapeutic Diabetes Education, Nagpur, India.

${ }^{2}$ Ex HOD RTMNU, Home Science, Nagpur, India.

${ }^{3}$ Sunil's Diabetes Care n' Research Centre Pvt Ltd., Daibetology, Nagpur, India.

\section{Background}

Hypertriglyceridemia is one of the risk factors for atherosclerosis in type 2 diabetes (T2DM). Post-prandial hypertriglyceridemia (PPTG) and hyperglycemia may jointly trigger spikes of oxidative stress causing undetermined risk of atherosclerosis. Conventionally, lipid profile is done in the fasting state, however post prandial increase in lipoprotein is inadequately addressed. India is facing a rapid transition of traditional balanced diet to high calorie, fat rich fast food culture.

\section{Aim}

To study the effect of standardized high fat, calorie dense Indian food on lipids and glucose amongst type 2 diabetics and non-diabetics.

\section{Materials And Methods}

After meeting the inclusion and exclusion criteria, 46 subjects were screened from a tertiary care diabetes centre of Central India for an interventional study, following the approval of an Institutional Ethic's committee. Finally, 44 subjects with 22 type 2 diabetics of more than six-month duration (Male-11, Female-11), non-alcoholic, $\mathrm{HbAlc}$ less than $8 \%$, not on lipid lowering medication (DM group) and 22 nondiabetics (Male-11, Female-11) non-alcoholic, not on lipid lowering medication, age and BMI matched (Non-DM group) were selected. Clinical history, examination, demographics and anthropometry data was collected. Baseline fasting (with 8-14 hours fast) blood sample (0hr) was collected for biochemical parameters. Postprandial lipid and glucose response was observed at $2 \mathrm{hr}$, $4 \mathrm{hr}$ and $6 \mathrm{hr}$ through fat tolerance test (FTT) using weight-adjusted Indian meal consisting of $15 \%$ saturated fatty acids, $55 \%$ monounsaturated fatty acids, $5 \%$ protein and $25 \%$ carbohydrates.

The statistical significance of difference of parameter between two groups at each time point and across time points was tested using t-test of independent samples and using repeated measure ANOVA respectively. The significance was tested at 5\% level.

\section{Conclusion}

- Postprandial hypertriglyceridemia occurs after ingestion of high fat meal in diabetics and non-diabetics. The increase is at it's peak at four hours followed by fall at $6 \mathrm{hrs}$.

- Type 2 diabetics showed significantly higher rise in PPTG at 4 hours and slower fall at 6 hours compared to non-diabetics.

- Post-prandial hyperglycemia at two hours and hypertriglyceridemia at fours might pose additive cardiovascular risk amongst Indian type 2 diabetics versus non-diabetics. Muticentric population specific studies on this concept are the need of the hour.

\section{Table \& Graphs}
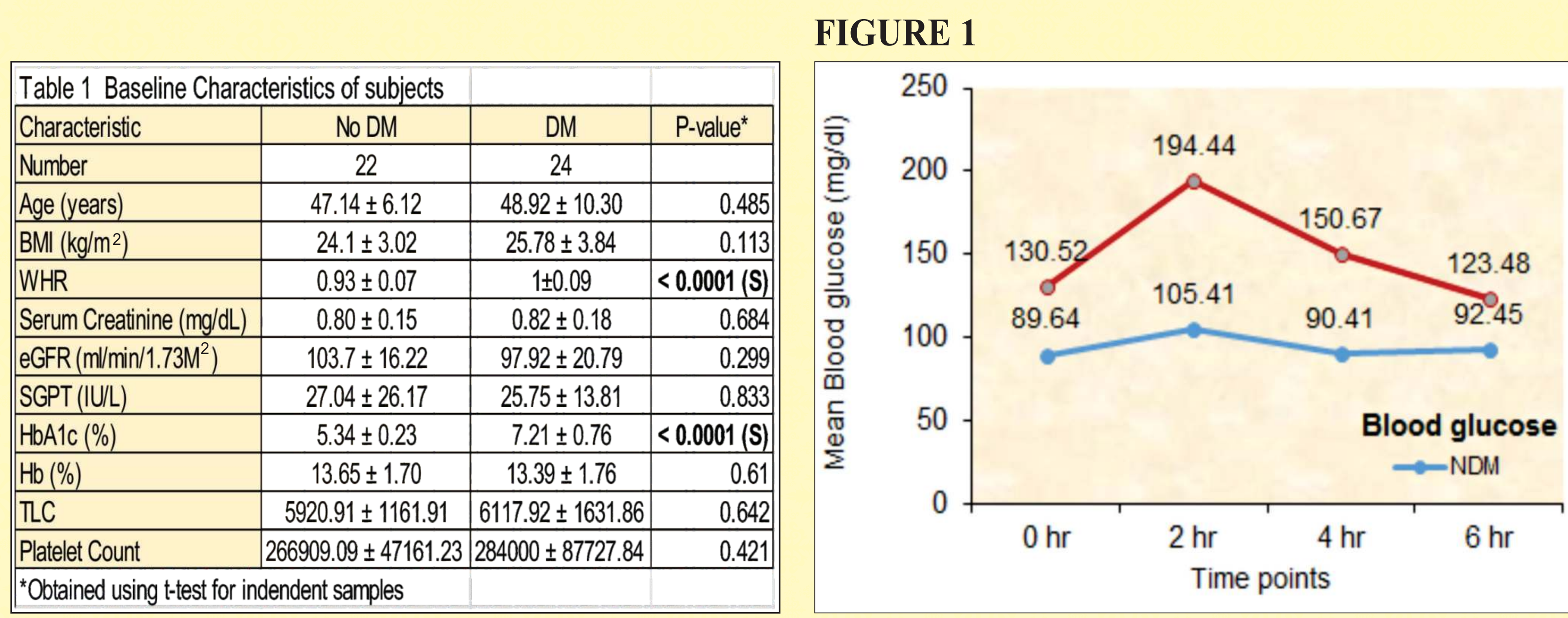

FIGURE 4

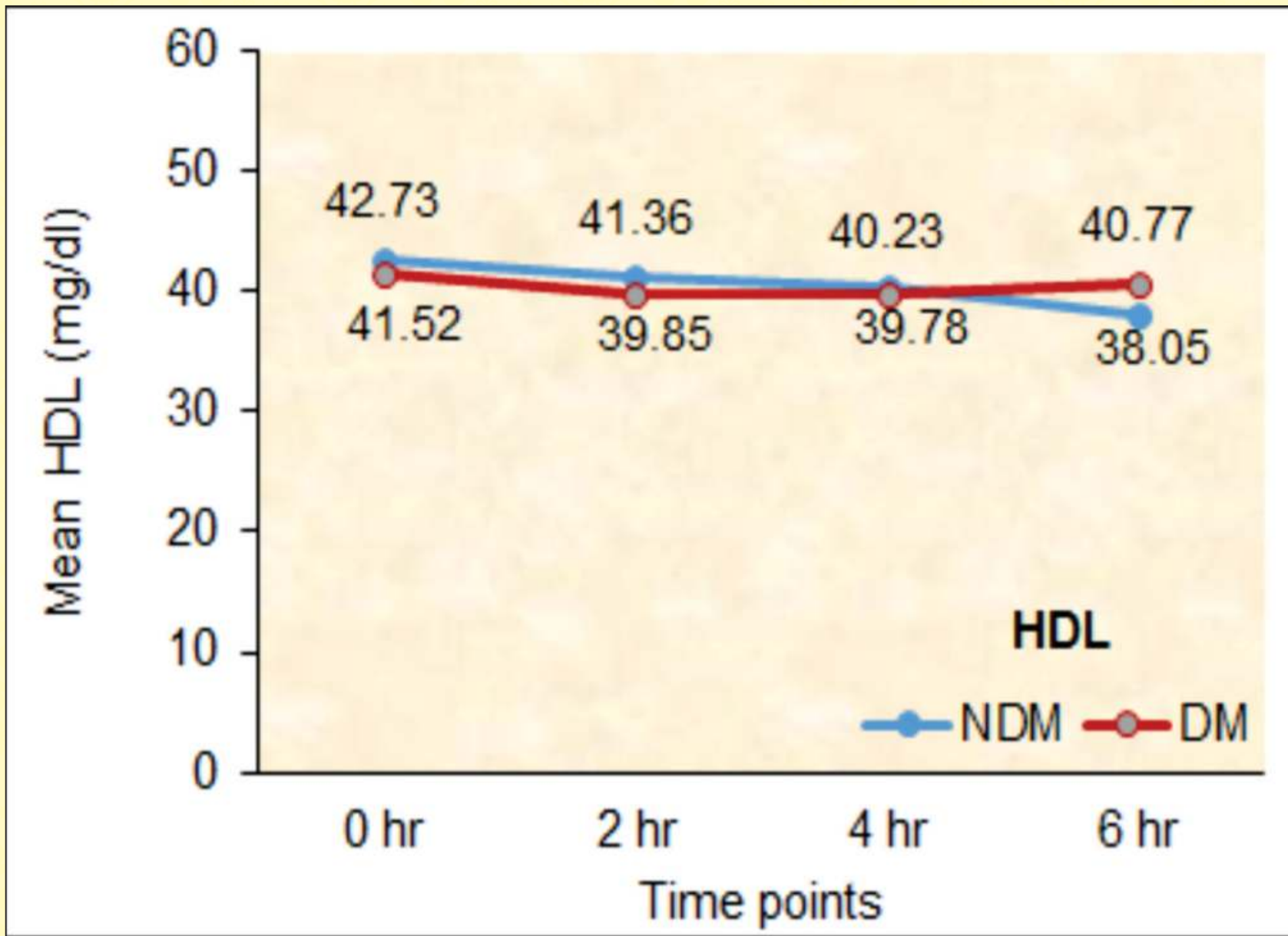

FIGURE 5

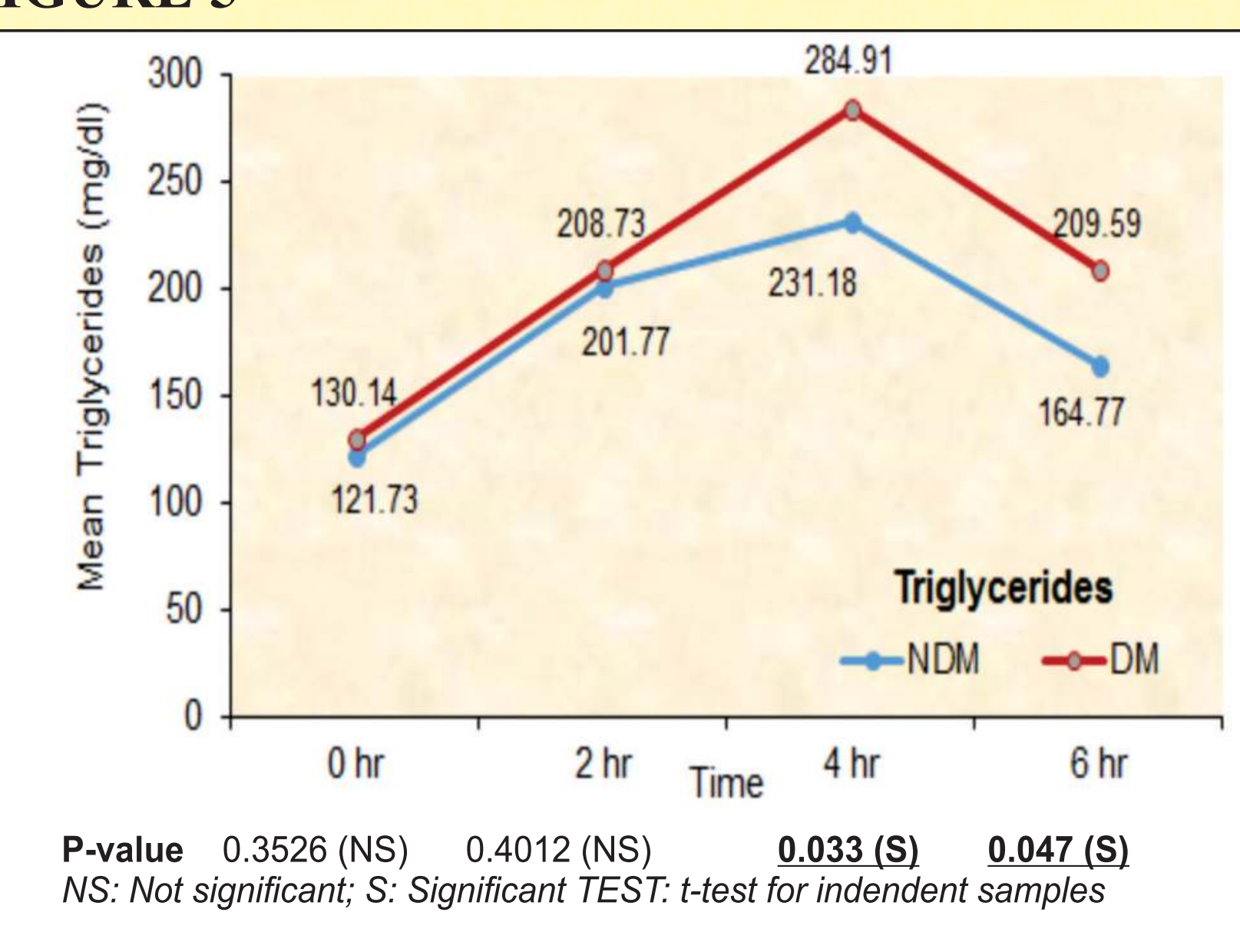

\section{Results}

- Age, BMI, S.Creatinine, eGFR were homogeneous across two groups. However, WHR, blood glucose, and HbAlc were significantly higher in DM group compared to Non-DM group $(\mathrm{P}<0.001)$. (Table 1 Figure 1)

- Mean blood glucose in DM group was significantly higher than Non DM group at baseline, $2 \mathrm{hr}$, $4 \mathrm{hr}$ and $6 \mathrm{hr}(\mathrm{P}<0.001)$. Mean total cholesterol (TC), LDL-C, HDL-C between two groups differed insignificantly at each time point $(\mathrm{P}>0.05)$ and when studied across times they revealed insignificant difference in both the groups. (Figure 2,3,4)

- Mean TG between two groups differed insignificantly at baseline and 2hr; however, at $4 \mathrm{hr}$ and $6 \mathrm{hr}$, TG was higher in DM versus Non-DM group, which was statistically significant with $\mathrm{P}=0.033$ and 0.047 respectively. (FIGURE 5)

- Across time points in Non DM group, the mean difference of TG was statistically significant with $\mathrm{P}<0.001$. Pair wise analysis in this group revealed that the difference between all the time pairs was statistically significant $(\mathrm{P}<0.05)$, except $2 \mathrm{hr}$ and $4 \mathrm{hr}(\mathrm{P}=0.127)$. In $\mathrm{DM}$ group, the mean difference of TG across times was significant with $\mathrm{P}<0.001$. Paired analysis in this group revealed that the difference between all the time pairs was significant, except between $2 \mathrm{hr}$ and $6 \mathrm{hr}(\mathrm{P}=0.99)$.

- In other words, in this group, at $6 \mathrm{hr}$, the mean TG level $(209.59 \mathrm{mg} / \mathrm{dl})$ was close to $2 \mathrm{hr}(208.73 \mathrm{mg} / \mathrm{dl})$, but still significantly higher than the baseline level (130.14 $\mathrm{mg} / \mathrm{dl})(\mathrm{P}<0.001)$ In Non-DM group also, the mean TG level at $6 \mathrm{hr}(164.77$ $\mathrm{mg} / \mathrm{dl}$ ) was significantly higher than baseline $(121.73 \mathrm{mg} / \mathrm{dl})$ level with $\mathrm{P}=0.005$; however, it was close to acceptable level of $150 \mathrm{mg} / \mathrm{dl}$, as compared to that of DM group. (FIGURE 6)

- Similarly, the mean change in TG between baseline and $4 \mathrm{hr}$ in Non DM group $(109.45 \mathrm{mg} / \mathrm{dl})$ differed significantly from that of DM group $(154.77 \mathrm{mg} / \mathrm{dl})$ with $\mathrm{P}=0.048$. (FIGURE 7)

- However, the mean change between $4 \mathrm{hr}$ and $6 \mathrm{hr}$ in Non DM group $(66.41 \mathrm{mg} / \mathrm{dl})$ insignificantly differed from that of DM group $(75.32 \mathrm{mg} / \mathrm{dl})$ with $\mathrm{P}=0.697$.
FIGURE 2

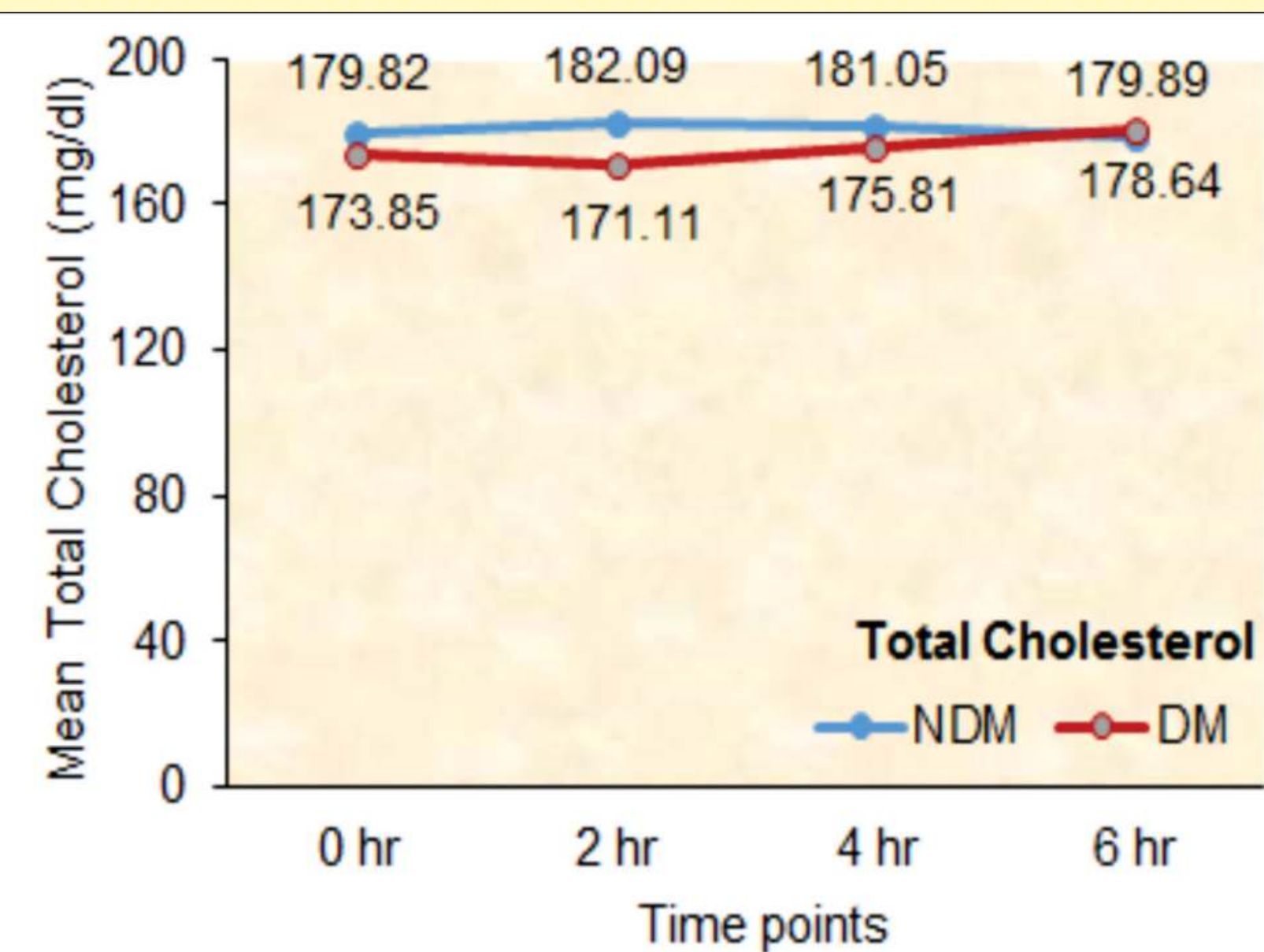

FIGURE 6

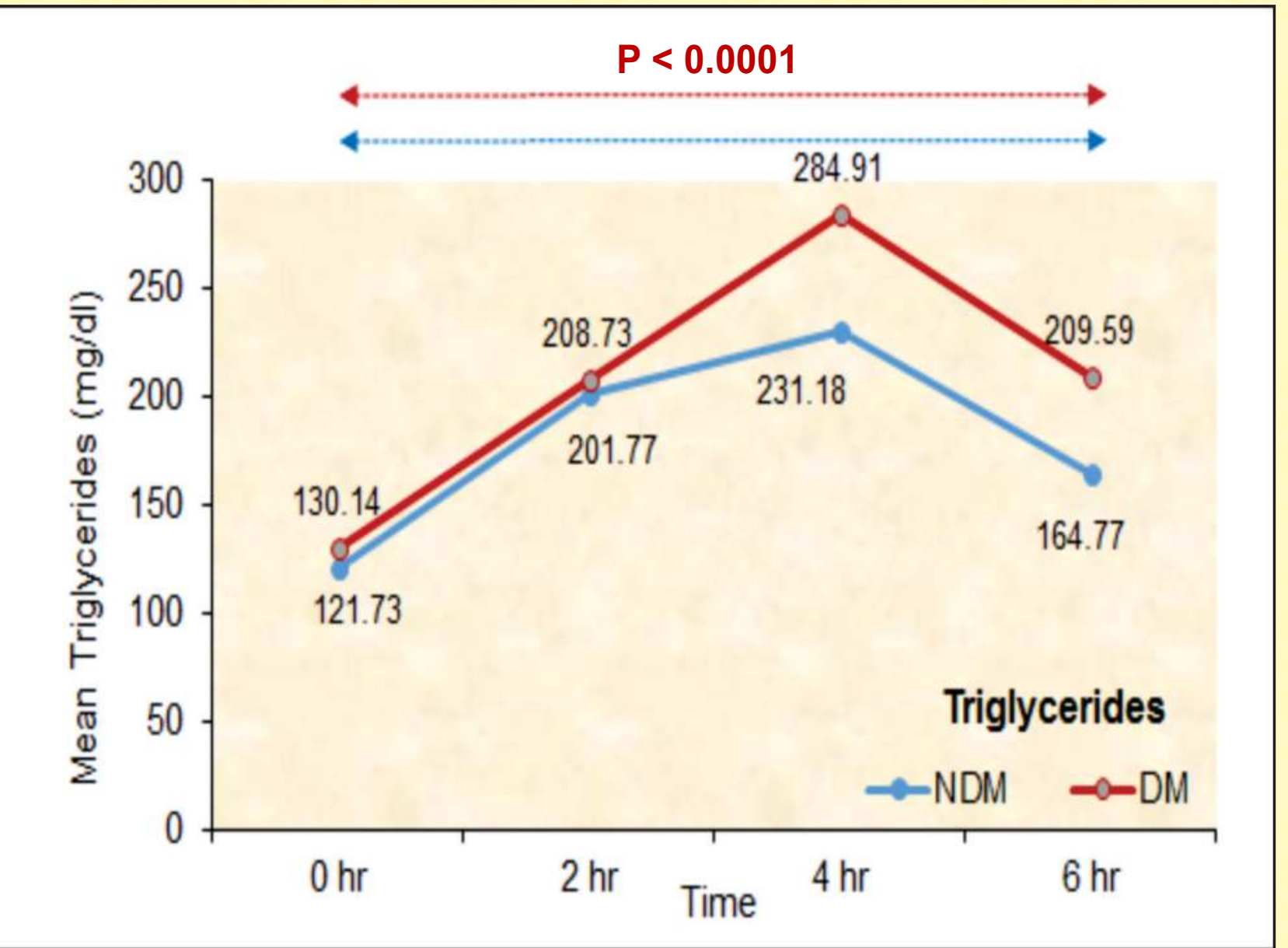

FIGURE 3

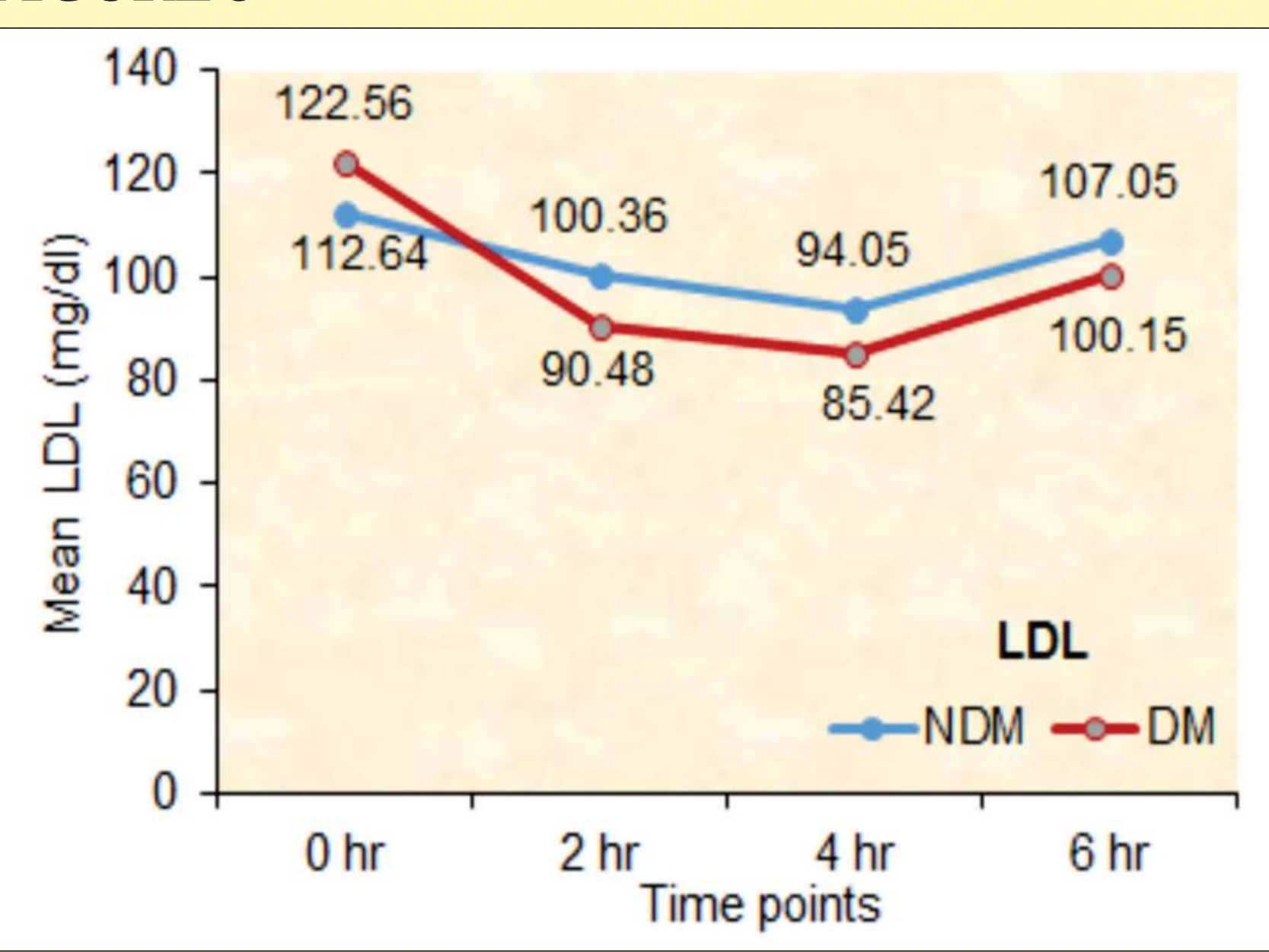

FIGURE 7

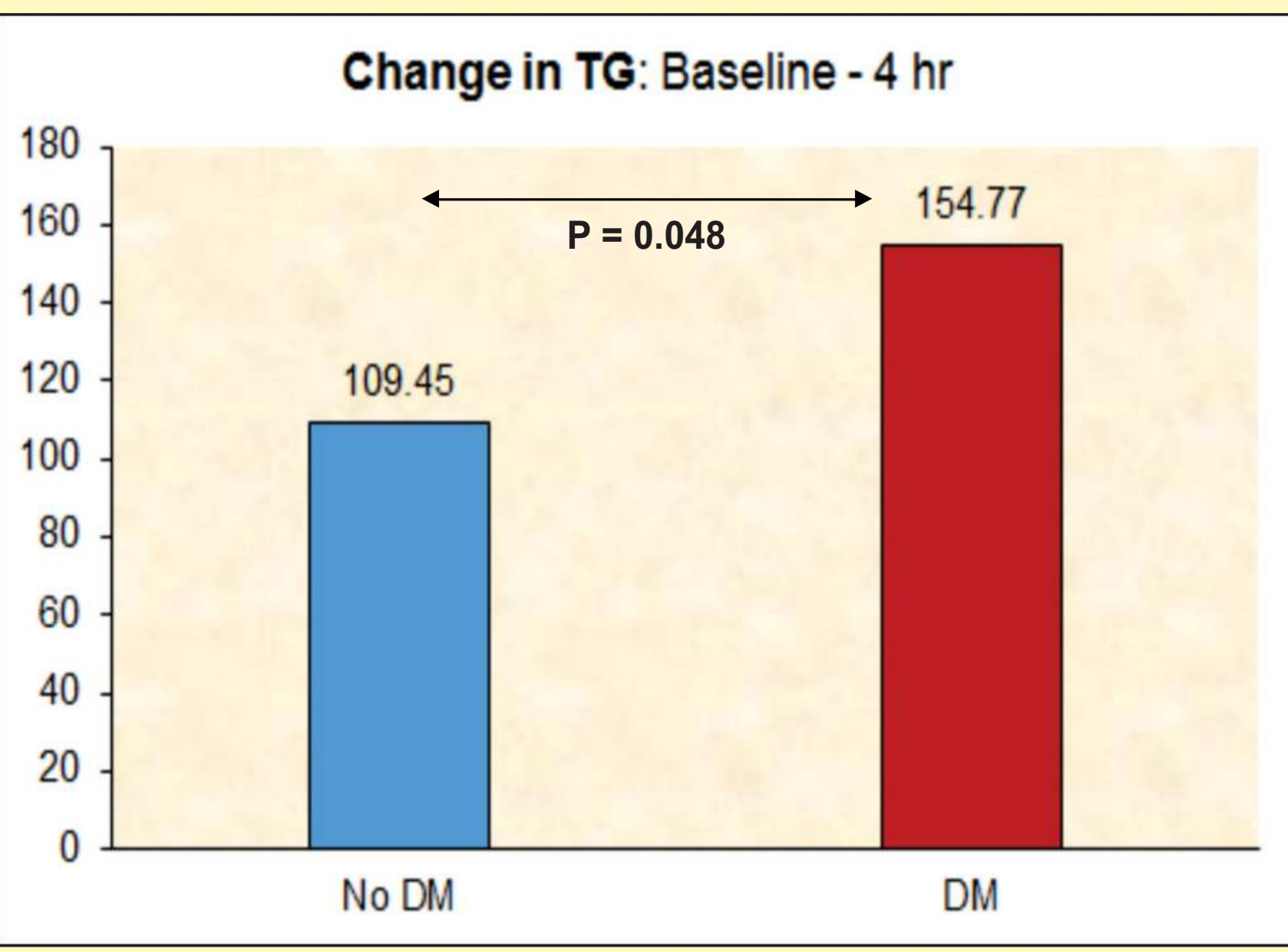

